\title{
Flora da Bahia: Boodleaceae
}

Aigara Miranda Alves ${ }^{1 *}$, Lísia Mônica de Souza Gestinari² \& Carlos Wallace do Nascimento Moura ${ }^{1}$

${ }^{1}$ Laboratório de Ficologia, Departamento de Ciências Biológicas, Universidade Estadual de Feira de Santana, Av. Transnordestina s/n, Bairro Novo Horizonte, 44036-900, Feira de Santana, Bahia, Brasil.

${ }^{2}$ Núcleo em Ecologia e Desenvolvimento Socioambiental de Macaé (NUPEM), Universidade Federal do Rio de Janeiro, Campus Macaé, C.P. 119331, 27910-970, Macaé, Rio de Janeiro, Brasil.

\begin{abstract}
Resumo - É apresentado o levantamento de Boodleaceae da Bahia, Brasil, como contribuição ao conhecimento das clorofíceas do litoral do estado. Foram reconhecidos três gêneros e quatro espécies: Boodlea composita, Cladophoropsis membranacea, Phyllodictyon anastomosans e P. pulcherrimum. É apresentada chave de identificação, além de descrições, ilustrações e comentários para os táxons e mapas de distribuição geográfica das espécies na Bahia.

Palavras-chave adicionais: Boodlea, Brasil, Chlorophyta, Cladophoropsis, Nordeste, Phyllodictyon.
\end{abstract}

\begin{abstract}
Flora of Bahia: Boodleaceae) - A survey of Boodleaceae from the coast of Bahia State, Brazil, is presented as a contribution to the knowledge of the chlorophytes. Three genera and four species were recognized: Boodlea composita, Cladophoropsis membranacea, Phyllodictyon anastomosans and P. pulcherrimum. An identification key, descriptions, illustrations and comments on taxa as well as distribution maps for the species in Bahia State are presented.

Additional key words: Boodlea, Chlorophyta, Cladophoropsis, Northeast Brazil, Phyllodictyon.
\end{abstract}

\section{Boodleaceae}

Talo filamentoso, unisseriado, filamentos livres ou entrelaçados por células tenaculares, com extremidades hapteroidais. Fixo ao substrato por rizoides ramificados, hapteroidais. Células multinucleadas. Cloroplastos formando uma rede parietal ou camada mais ou menos contínua, pirenoides presentes. Crescimento do talo por divisão de células apicais e intercalares. Divisão celular por invaginação centrípeta da parede; ocasionalmente ocorre divisão segregativa modificada, devido a dano celular, na qual as massas citoplasmáticas formadas, após secretarem novas paredes celulares, são liberadas da célula parental, desenvolvendo-se em novos talos. Reprodução assexuada por zoósporos biflagelados e por aplanósporos, produzidos por divisão celular segregativa. Histórico de vida haplodiplonte e isomórfico. Gametófito produzindo gametas biflagelados; esporófito produzindo meiósporos quadriflagelados.

Boodleaceae inclui cinco gêneros (Boodlea, Cladophoropsis, Phyllodictyon, Struvea Sonder e Symbiomonas Guillou \& M.J.Chrétiennot-Dinet) e 30 espécies, nos oceanos Atlântico, Índico e Pacífico (Guiry \& Guiry 2012). Todos os gêneros, exceto Symbiomonas, são registrados para o Atlântico Americano, totalizando oito espécies (Wynne 2011), seis registradas para o litoral do Brasil (Moura et al. 2012).

\section{Chave de identificação}

1. Talos estipitados, filamentos formando lâmina reticu-

*Autora para correspondência: aigarama@yahoo.com.br

Editor responsável: Alessandro Rapini

Submetido em: 15 ago. 2012; publicação eletrônica: 21 dez. 2012 lada anastomosada por células tenaculares apicais e laterais. 3. Phyllodictyon

2. Talo até $4 \mathrm{~cm}$ alt., portando células com inclusões cristalinas em forma de hexágonos alongados a finas agulhas agrupadas; células apicais 250-381-550 $\mu \mathrm{m}$ compr. $\times 130-156-210 \mu \mathrm{m}$ diâm.3.1. P. anastomosans

2'. Talo 7-10 cm alt., portando células com inclusões cristalinas em forma de hexágonos largos; células apicais 450-997-2300 $\mu \mathrm{m}$ compr. $\times 190-258-320$ um diâm 3.2. P. pulcherrimum

1'. Talos sem estipe, filamentos não formando lâmina.

3. Ramificação oposta, células apicais 380-1198$11360 \mu \mathrm{m}$ compr. $\times 100-128-160 \mu \mathrm{m}$ diâm.

1.1. Boodlea composita

3'. Ramificação irregular, células apicais 880-1277$2660 \mu \mathrm{m}$ compr. $\times 100$-151-260 $\mu \mathrm{m}$ diâm. .2.1. Cladophoropsis membranacea

\section{Boodlea (Dickie) G.Murray \& De Toni}

Talo composto por filamentos unisseriados, irregularmente ramificados, crescendo em forma de almofadas esponjosas, de forma indefinida. Fixo ao substrato por rizoides ramificados originados da célula de talos jovens, por rizoides produzidos no ápice das células apicais e por células tenaculares originadas nas extremidades distal e lateral das células. Ramos laterais com atraso na formação de septos, permanecendo conectados à célula parental. Reforço estrutural do talo por entrelaçamento de ramos curvos e anastomoses de células adjacentes, por células tenaculares. Inclusões cristalinas birrefringentes de oxalato de cálcio, em forma de hexágonos alongados a finas agulhas agrupadas.

O gênero possui distribuição pantropical, ocorrendo nos Sitientibus série Ciências Biológicas 12(2): 179-188. 2012. 
oceanos Atlântico, Pacífico e Índico. De acordo com Guiry \& Guiry (2012), sete espécies são reconhecidas, duas destas, Boodlea composita e B. struveoides M.A.Howe, referidas para o Atlântico Americano (Wynne 2011) e apenas a primeira referida para o Brasil (Moura et. al 2012).

1.1. Boodlea composita (Harvey) F.Brand, Beih. Bot. Centralbl. 18(1): 165; pl. 6, fig. 28-35. 1904. Conferva composita Harvey, J. Bot. (Hooker) 1: 157. 1834.

Figuras 1 e 2.

Talo verde-claro, prostrado a ereto, formando densas almofadas, composto de filamentos ramificados. Ramificação oposta a unilateral; eixo principal com ramificação oposta na base a irregular na região mediano-apical, com divisões celulares intercalares frequentes. Célula apical cilíndrica, ápice arredondado, reto ou curvado, 380-1198-11360 $\mu \mathrm{m}$ compr. $\times 100-128-160 \mu \mathrm{m}$ diâm.; células do eixo principal cilíndricas, 300-592-1300 $\mu \mathrm{m}$ compr. $\times$ 90-135-200 $\mu \mathrm{m}$ diâm.; parede celular $>2,5 \mu \mathrm{m}$ espessura. Inclusões cristalinas birrefringentes de oxalato de cálcio, em forma de hexágonos alongados a finas agulhas agrupadas, presentes em todas as células do talo, exceto nas células tenaculares. Células férteis com protoplasto dividido, com estruturas reprodutivas liberadas por papilas laterais e apicais.

Boodlea composita é amplamente distribuída nos oceanos Atlântico, Índico e Pacífico (Guiry \& Guiry 2012). No litoral brasileiro, a espécie é registrada apenas para os estados de Pernambuco, Bahia e Rio de Janeiro (Yoneshigue et al. 1986; Alves et al. 2012; Soares \& Fujii 2012). D10, E/ F9, G8/9, K8: sobre substrato rochoso, formando tufos densos, em locais protegidos ou expostos à arrebentação das ondas, na região entremarés. Talos férteis encontrados nos meses de março e junho.

Material selecionado - Conde, Sítio do Conde, 20 abr. 2007, A.M. Alves \& C.S. Santana s.n. (HUEFS 136300); Ilha de Itaparica, Vera Cruz, Praia da Conceição, 18 maio 2007, A.M. Alves et al. s.n. (HUEFS 136304); Ilhéus, Praia do Aeroporto, 8 set. 2006, A.M. Alves et al. s.n. (HUEFS 136307); Mucuri, Praia da Costa Dourada, 12 jun. 2007, A.M. Alves \& C.S. Santana s.n. (HUEFS 136308); Salvador, Praia da Pituba, 9 ago. 2006, A.M. Alves s.n. (HUEFS 136301).

Material adicional examinado - BRASIL. RIO DE JANEIRO: Ponta Pai Vitório, Mesolitoral, 28 jul. 1983, Y. Yoneshigue, YY 4010H (RFA); 18 jul. 1981, Y. Yoneshigue, MOF 352 (RFA); 18 jul. 1981, Y. Yoneshigue, YY 2831 (RFA).

Cresce associada a Anadyomene stellata (Wulfen) C.Agardh, Boodleopsis pusilla (F.S.Collins) W.R.Taylor, A.B.Joly \& Bernatowicz, Bryopsis pennata J.V.Lamouroux, Chaetomorpha brachygona Harvey, Cladophora vagabun$d a$ (L.) C.Hoek, Cladophoropsis membranacea (H.Bang ex C.Agardh) Børgesen, Caulerpa fastigiata Montagne, Caulerpella ambigua (Okamura) Prud'homme \& Lokhorst, Dictyosphaeria versluysii Weber Bosse, Phyllodictyon anastomosans (Harvey) Kraft \& M.J.Wynne, Dictyopteris delicatula J.V.Lamouroux, Amphiroa anastomosans Webervan Bosse, A. fragilissima (L.) J.V.Lamouroux, Digenea

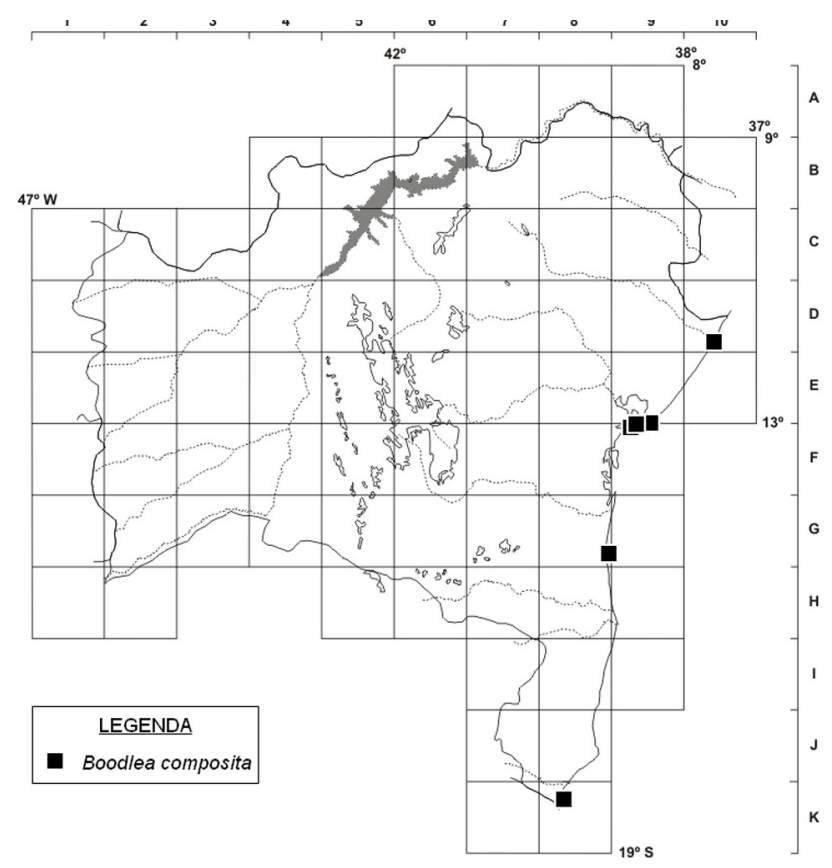

Figura 1. Mapa de distribuição de Boodlea composita no litoral do estado da Bahia.

simplex (Wulfen) C.Agardh, Hypnea musciformis (Wulfen) J.V.Lamouroux, Jania adhaerens J.V.Lamouroux e Palisada perforata (Bory de Saint-Vincent) K.W.Nam. É epifitada por Erythrotrichia carnea (Dillwyn) J.Agardh, Sahlingia subintegra (Rosenv.) Kornmann, calcárias crostosas e diatomáceas.

\section{Cladophoropsis Børgesen}

Talo ereto a prostrado, composto por filamentos entrelaçados crescendo em forma de almofada. Fixo ao substrato por rizoides multicelulares, originados da base da célula basal, por rizoides formados em qualquer parte do talo ou por células tenaculares. Crescimento do talo por divisão celular apical com subsequente elongação celular e por divisão celular intercalar na parte basal do talo. Divisão celular por invaginação centrípeta da parede e, ocasionalmente, por divisão segregativa modificada. Ramificação irregular, com pronunciado atraso na formação de septo na base dos ramos. Reforço estrutural do talo por entrelaçamento dos filamentos, em muitas espécies por anastomoses de filamentos adjacentes por células tenaculares. Células multinucleadas com cloroplastos poligonais unidos formando uma rede parietal com numerosos pirenoides. Inclusões cristalinas birrefringentes de oxalato de cálcio presentes no protoplasto. Estruturas reprodutivas liberadas por papilas formadas nas laterais das células. Propagação vegetativa por aplanósporos produzidos por divisão celular segregativa.

$O$ gênero ocorre em ambientes marinho e estuarino com ampla distribuição nos oceanos Atlântico, Índico e Pacífico. Segundo Guiry \& Guiry (2012), Cladophoropsis apresenta nove espécies. Dentre estas, $C$. macromeres Taylor, $C$. 

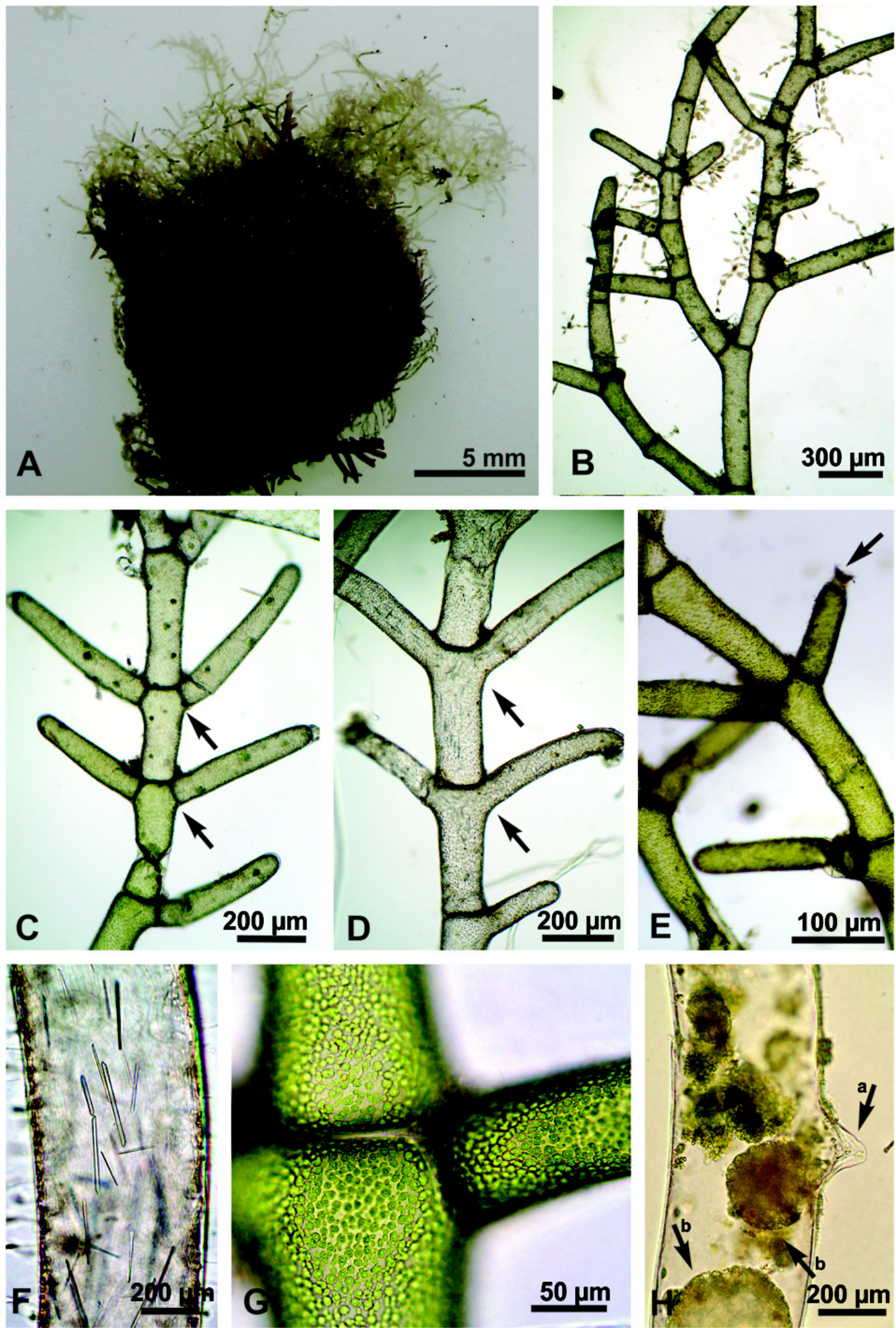

Figura 2. Boodlea composita: A- aspecto geral; B- detalhe da ramificação do talo; C- ramificação oposta com ramos septados (setas); D- ramificação oposta com ramos asseptados (setas); E- detalhe de célula tenacular (seta); F- cristais de oxalato de cálcio em forma de hexágonos alongados; G- detalhe dos cloroplastos formando retículo; H- célula fértil com papila (a) e massas citoplamáticas formadas por divisão celular segregativa (b) (A, C, E- HUEFS 136302; B- HUEFS 136301; D- HUEFS 136307; F- HUEFS 136300; G- HUEFS 136306; H- HUEFS 136308). 
membranacea (H.Bang ex C.Agardh) Børgesen e $C$. fasciculata (Kjellman) Wille ocorrem no Atlântico Americano (Wynne 2011), as duas primeiras com registro para o Brasil (Széchy et al. 1987; Pedrini et al. 1992; Moura et al. 2012).

2.1. Cladophoropsis membranacea (H.Bang ex C.Agardh) Børgesen, Overs. Kongel. Danske Vidensk. Selsk. Forh. 3: 289, legend to fig. 8-13. 1905. Conferva membranacea H.Bang ex C.Agardh, Syst. Alg.: 120. 1824. Figuras 3 e 4.

Talo verde-claro a verde-escuro, composto de filamentos fortemente entrelaçados, por células tenaculares, consistência firme, formando tufos densos e extensos tapetes em forma de almofada, até $3,5 \mathrm{~cm}$ alt. Ramificação unilateral a irregular, até $3^{\mathrm{a}}$ ordem, filamentos asseptados, secundariamente septados na base. Células apicais cilíndricas, eretas a curvadas, dimensões 800-1554-2180 $\mu \mathrm{m}$ compr. $\times 100$ 151-260 $\mu \mathrm{m}$ diâm.; células do eixo principal com 4501153-2400 $\mu$ m compr. $\times 80-112-190 \mu$ m diâm. Células dos eixos secundários com 880-1277-2660 $\mu \mathrm{m}$ compr. $\times 60$ 91-180 $\mu \mathrm{m}$ diâm. Parede celular 10-20 $\mu \mathrm{m}$ espessura. Não foram observadas inclusões cristalinas nas células. Células férteis com papilas laterais e apicais para liberação das estruturas reprodutivas.

Espécie amplamente registrada nos oceanos Atlântico, Índico e Pacífico (Guiry \& Guiry 2012). No litoral do Brasil, a espécie é registrada para os estados do Maranhão, Ceará, Rio Grande do Norte, Paraíba, Pernambuco, Alagoas, Bahia, Espírito Santo, Rio de Janeiro, São Paulo, Paraná, Santa Catarina e para o Arquipélago de Fernando de Noronha e Ilha de Trindade (Széchy et al. 1987; Pedrini et al. 1992; Moura et al. 2012). D10, E9, E/F9, E10, F9, G8/9, G/H8, I8/9, K8: comum na área estudada, formando densos e extensos tapetes em costões rochosos, descobertos nas marés baixas, em locais protegidos da arrebentação e sobre pneumatóforos em manguezal. Talos férteis encontrados em março.

Material selecionado - Conde, Sítio do Conde, 20 abr. 2007, A.M. Alves \& C.S. Santana s.n. (HUEFS 136594); Entre Rios, Subaúma, 19 abr. 2007, A.M. Alves \& C.S. Santana s.n. (HUEFS 136593); Ilhéus, Praia de Back Door, 8 set. 2006, A.M. Alves et al. s.n. (HUEFS 136615); Itacaré, Praia da Concha, 9 set. 2006, A.M. Alves et al. s.n. (HUEFS 136616); Madre de Deus, Mangue do Cação, 6 abr. 2007, G.L. Alves s.n. (HUEFS 136507); Maragogipe, 19 mar. 2007, A.M. Alves et al. s.n. (HUEFS 136610); Mucuri, Praia da Costa Dourada, 12 jun. 2007, A.M. Alves \& C.S. Santana s.n. (HUEFS 136618); Salinas da Margarida, 19 mar. 2007, A.M. Alves et al s.n. (HUEFS 136611); Cairu, Ilha de Boipeba, Praia da Ponta dos Castelhanos, 4 jul. 2007, C.W.N. Moura et al. s.n. (HUEFS 136613); Salvador, Praia do Rio Vermelho, 15 out. 1997, S.A. Silva \& M.C. Accioly s.n. (ALCB 036792); Santa Cruz Cabrália, Arakakaí, 12 jul. 2006, C.W.N. Moura et al. s.n. (HUEFS 136617); Santo Amaro, Praia de Itapema, 17 mar. 2007, A.M. Alves et al. s.n. (HUEFS 136508); São Francisco do Conde, Ilha de Cajaíba, 16 mar. 2007, A.M. Alves \& C.S. Santana s.n. (HUEFS 136506); Saubara, 17 mar. 2007, A.M. Alves et al. s.n. (HUEFS

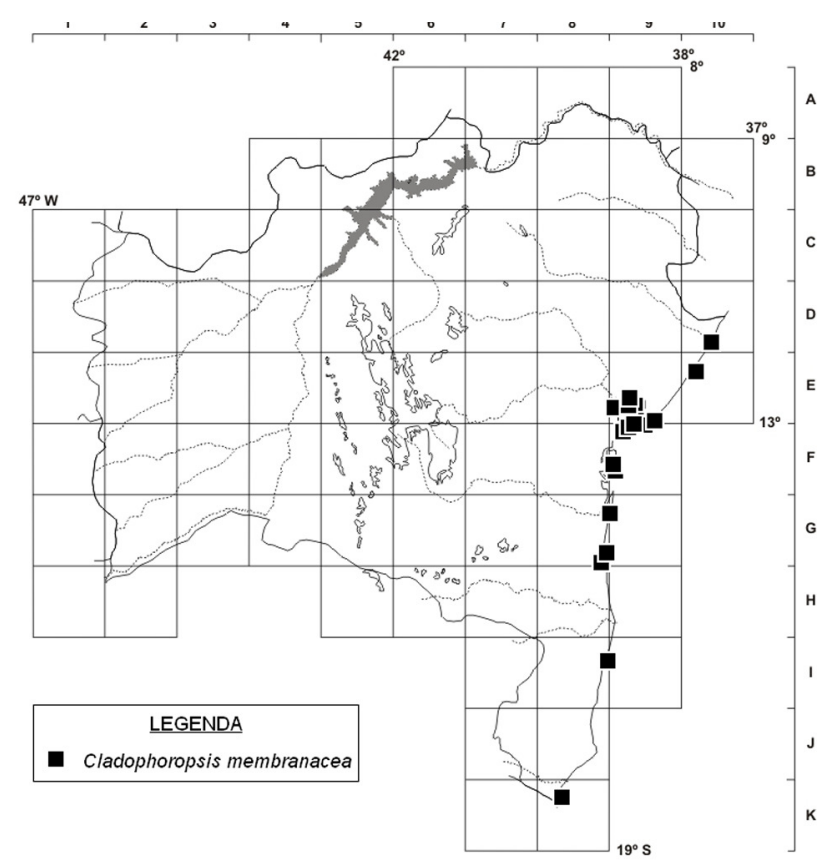

Figura 3. Mapa de distribuição de Cladophoropsis membranacea no litoral do estado da Bahia.

136509); Ilha de Itaparica, Vera Cruz, Praia de Cacha-Pregos, manguezal, 19 mar. 2007, A.M. Alves et al. s.n. (HUEFS 136605).

Cladophoropsis membranacea tem sido confundida com C. fasciculata e C. macromeres devido às dimensões celulares intermediárias. Segundo Leliaert \& Coppejans (2006), C. macromeres difere de C. membranacea por apresentar filamentos mais espessos: células apicais (140-)280-360(400) $\mu \mathrm{m}$, laterais $220-300 \mu \mathrm{m}$ e as do eixo principal 280 $510 \mu \mathrm{m}$; enquanto $C$. fasciculata difere de $C$. membranacea por possuir filamentos mais finos: células apicais (40-)60$120(-140) \mu \mathrm{m}$, terminais $80-140 \mu \mathrm{m}$ e basais $80-180-250$ $\mu \mathrm{m}$.

Células férteis de Cladophoropsis membranacea com papilas para liberação das estruturas reprodutivas e células em processo de divisão celular segregativa modificada estão sendo registradas pela primeira vez para o litoral brasileiro. A espécie cresce associada a Boodlea composita, Boodleopsis pusilla, Caulerpa fastigiata, Cladophora catenata (L.) Kütz. emend. C.Hoek, Chaetomorpha aerea (Dillwyn) Kütz., C. brachygona, C. vieillardii (Kütz.) M.J.Wynne, Rhizoclonium riparium (Roth) Kütz. ex Harv., Dictyopteris polypodioides (DC.) J.V.Lamouroux, Acanthophora muscoides (L.) Bory de Saint-Vincent, A. spicifera (Vahl) Børgesen, Bostrychia radicans (Montagne) Montagne, B. tenella (J.V. Lamouroux) J.Agardh, Caloglossa leprieurii (Montagne) G.Martens, Ceramium dawsonii A.B.Joly, Corallina panizzoi R.Schnetter \& U.Richter, Gelidiella acerosa (Forsskal) Feldmann \& Hamel, Murrayella periclados (C.Agardh) F.Schmidt, Palisada perforata e Polysiphonia subtilissima Mont. Epifitada por Herposiphonia secunda (C.Agardh) Ambronn, H. secunda f. tenella (C.Agardh) M.J.Wynne e Erythrotrichia carnea. 

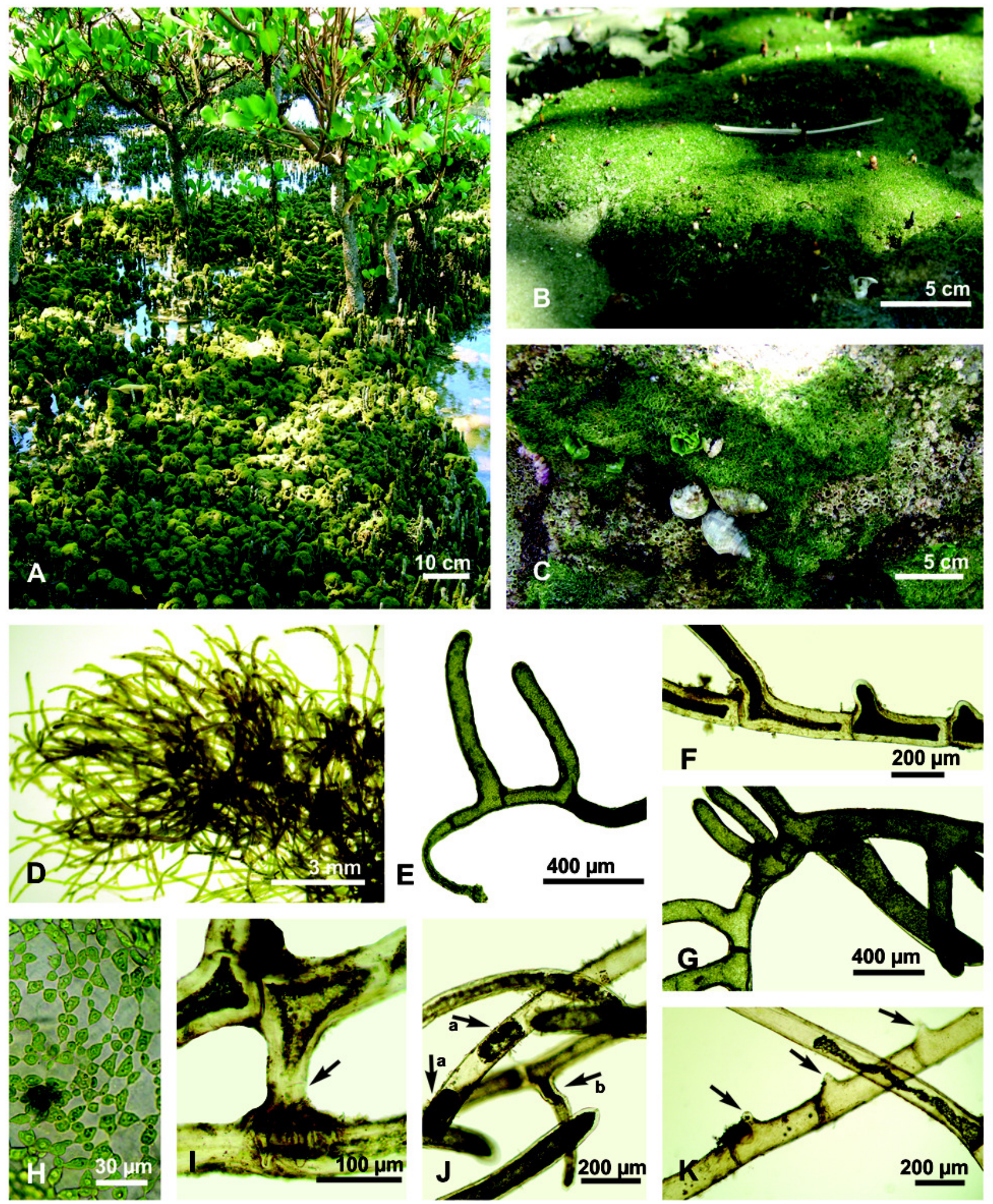

Figura 4. Cladophoropsis membranacea: A-C- hábito (A- tufos formando agregados densos sobre pneumatóforos; B- tufos formando tapetes em sedimento lodoso de manguezal; C- tufos crescendo sobre rocha de arenito); D-F- ramificação unilateral do talo com células asseptadas; G- detalhe do formato típico do talo; H- detalhe do talo mostrando cloroplastos formando retículo; I- célula tenacular (seta); J- massas citoplasmáticas, formadas por divisão celular segregativa modificada (a); note rizoide (b); K- ramos com células férteis com papilas (setas) (A- HUEFS 136595; B- HUEFS 136508; C- HUEFS 136594; D- HUEFS 136605; E- HUEFS 136506; F- HUEFS 136616; G- HUEFS 166506; H- HUEFS 136617; I, J- HUEFS 136593; KHUEFS 136610) 


\section{Phyllodictyon J.E.Gray}

Talo ereto, formando lâmina estipitada, com aspecto de rede, composta de filamentos densamente ramificados. Fixo ao substrato por rizoides multicelulares originados na base do estipe. Estipe simples ou ramificado, com ou sem constricções anelares. Lâmina formada por repetidos processos de divisão celular intercalar, através de invaginação centrípeta da parede. Ramificação oposta, num mesmo plano, até $3^{a}$ ordem. Divisão celular intercalar ocorrendo em intervalos regulares, da $3^{\mathrm{a}}$ a $7^{\mathrm{a}}$ célula abaixo da célula apical, ocasionalmente ocorre divisão celular segregativa modificada. Reforço estrutural da lâmina resultante da união de filamentos adjacentes por células tenaculares, formadas no ápice e lateral das células ou formadas intracelularmente entre células vizinhas de um mesmo filamento. Células multinucleadas, com cloroplastos poligonais a arredondados, unidos formando uma rede aberta ou um retículo parietal mais ou menos fechado, cada um com um único pirenoide. Inclusões cristalinas birrefringentes de oxalato de cálcio, em forma de hexágonos alongados a finas agulhas agrupadas e de hexágonos largos, nas células da lâmina, exceto nas células tenaculares.

Phyllodictyon é exclusivamente marinho, com distribuição nas águas tropicais e temperadas dos oceanos Atlântico, Índico e Pacífico. Está representado por nove espécies (Guiry \& Guiry 2012), duas referidas para o Atlântico Americano (Wynne 2011) e para o litoral do Brasil (Széchy et al. 1987; Nassar 1994; Moura et al. 2012).

3.1.Phyllodictyon anastomosans (Harvey) Kraft \& M.J.Wynne, Phycol. Res. 44: 139; fig. 16-25. 1996. Cladophora anastomosans Harvey, Phycol. Austral. 2: pl. CI. 1859.

Figuras 5 e 6.

Talo verde-claro a verde-escuro, ereto a curvado, formando lâmina estipitada, crescendo em tufos densos, até 4 $\mathrm{cm}$ alt. Fixo ao substrato por rizoides ramificados e septados, originados do pólo proximal do estipe. Ramificação oposta, em um único plano, resultando em lâminas aplanadas, até $11^{\mathrm{a}}$ ordem. Estipe ramificado ou não, sem constricções anelares basais, formado por célula longa, cilíndrica a clavada, 0,7-0,9-1,2 cm compr. × 330-571-700 $\mu \mathrm{m}$ diâm. Lâmina reticulada e achatada, $0,5-3 \mathrm{~cm}$ diâm., composta de filamentos densamente ramificados. Reforço estrutural do talo através de células tenaculares presentes no ápice $\mathrm{e}$ lateral das células. Células apicais com ápice arredondado, reto ou levemente curvado, 250-381-550 $\mu \mathrm{m}$ compr. $\times 130$ 156-210 $\mu \mathrm{m}$ diâm.; células do eixo principal 480-1066$1780 \mu \mathrm{m}$ compr. $\times 210-410-720 \mu \mathrm{m}$ diâm.; parede celular $>2,5 \mu \mathrm{m}$ espessura nas células apicais, 5-20 $\mu \mathrm{m}$ espessura nas do eixo principal. Cristais birrefringentes de oxalato de cálcio, em forma de hexágonos alongados a finas agulhas agrupadas, presentes em todas as células do talo. Células férteis com protoplasto segregado, com estruturas reprodutivas liberadas por papilas (1-3) laterais.

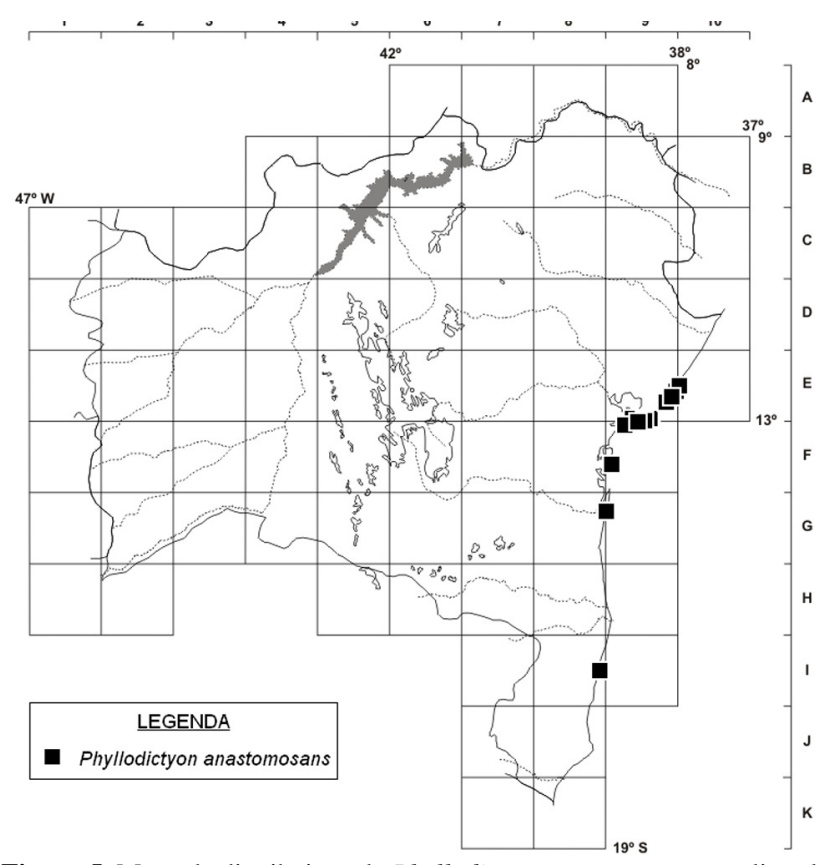

Figura 5. Mapa de distribuição de Phyllodictyon anastomosans no litoral do estado da Bahia

Phillodictyon anastomosans é amplamente distribuída nos oceanos Atlântico, Índico e Pacífico (Guiry \& Guiry 2012). No litoral brasileiro, é registrada para os estados do Ceará, Rio Grande do Norte, Paraíba, Pernambuco, Alagoas, Bahia, Espírito Santo e para o Arquipélago de Fernando de Noronha e Ilha de Trindade (Széchy et al. 1987; Muniz 1993; Nassar 1994; Moura et al. 2012). E9/10, E/F9, F9, G8/9, I8: comum na área estudada, crescendo na região entremarés, sobre substrato rochoso de platô recifal e em áreas sombreadas e protegidas da arrebentação, formando tufos frouxos e grandes, ou, mais frequentemente, em áreas de arrebentação, formando tufos pequenos densamente agregados. Talos férteis encontrados em agosto.

Material selecionado - Cairu, Ilha de Boipeba, Praia de Bainema, 2 jul. 2007, C.W.N. Moura et al. s.n. (HUEFS 136326); Camaçari, Arembepe, Praia de Piruí, 17 fev. 2007, A.M. Alves \& C.S. Santana s.n. (HUEFS 136311); Itha de Itaparica, Vera Cruz, Praia da Barra Grande, 18 maio 2007, A.M. Alves et al. s.n. (HUEFS 136322); Itacaré, Praia da Concha, 9 set. 2006, A.M. Alves et al. s.n. (HUEFS 136328); Mata de São João, Praia do Forte, 11 ago. 2006, A.M. Alves et al. s.n. (HUEFS 136309); Porto Seguro, Arraial d'Ajuda, Praia de Mucugê, 13 jul. 2006, C.W.N. Moura et al. s.n. (HUEFS 136329). Salvador, Praia de Itapuã, 12 ago. 2006, A.M. Alves et al. s.n. (HUEFS 136312).

Phyllodictyon anastomosans difere de P. pulcherrimum por apresentar talos menores e células com cristais de oxalato de cálcio em forma de hexágonos alongados a finas agulhas agrupadas. Além disso, P. pulcherrimum é tida como uma espécie de águas profundas. Kanagawa (1984) fez a primeira referência sobre a presença de cristais laminares, estreitos, afilados em ambas as extremidades (aciculares em perfil) para o Brasil, porém não relatou a natureza química. Estes cristais são semelhantes aos observados no material presen- 

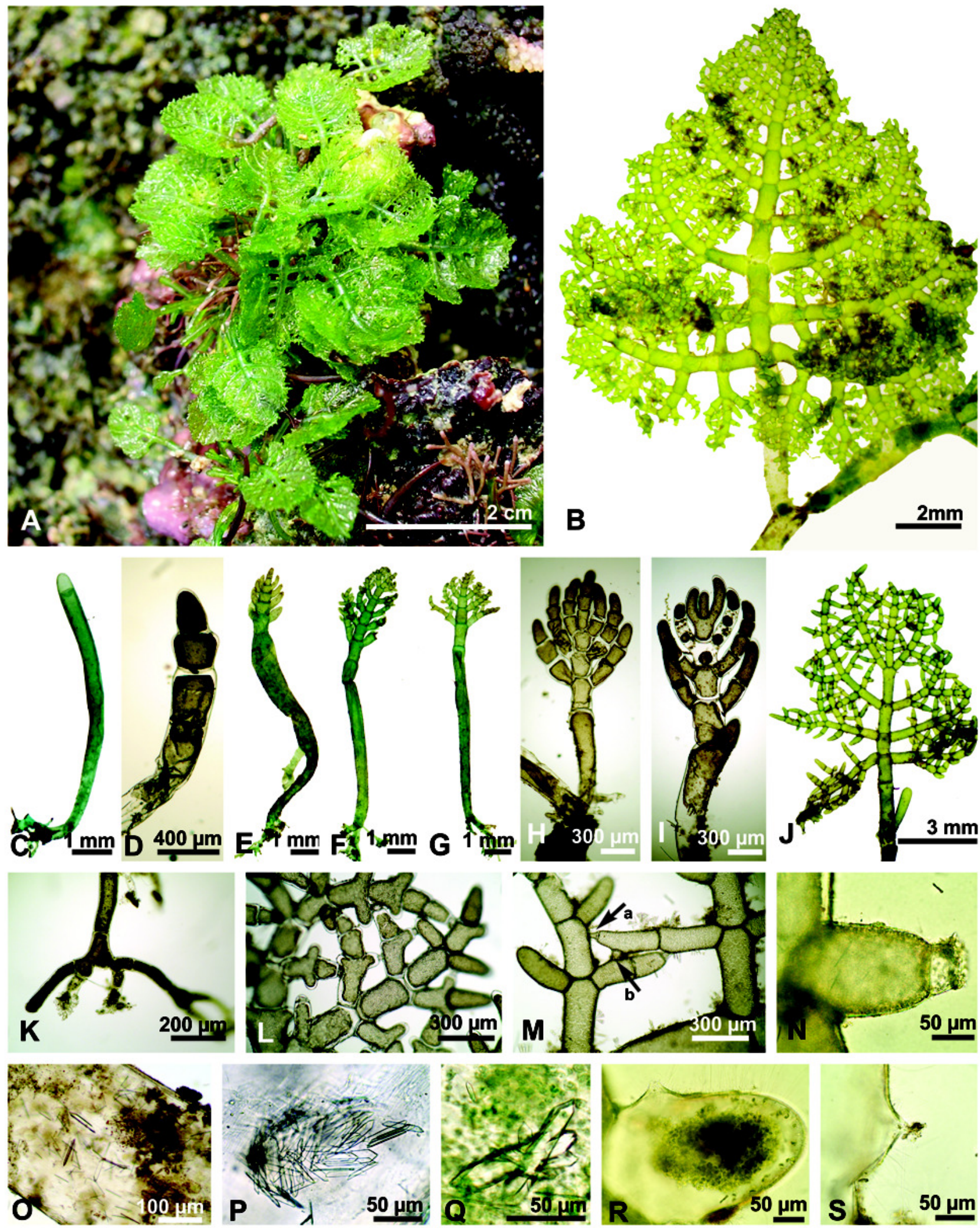

Figura 6. Phyllodictyon anastomosans: A- hábito; B- detalhe da lâmina com ramificação oposta; C-J- difentes estádios de desenvolvimento do talo; Kbase do estipe com rizoides; L- detalhe da lâmina formada por união de células tenaculares; M- detalhe de células tenaculares formadas no ápice (a) e lateral (b) das células; $\mathbf{N}$ - detalhe de célula tenacular; $\mathbf{O}-\mathbf{Q}$ - cristais de oxalato de cálcio em forma de hexágonos finos alongados a finas agulhas agrupadas; R- célula apical fértil com papila; S- detalhe da papila (A, B- HUEFS 136315; C-J- HUEFS 136320; K- HUEFS 136328; L, M- HUEFS 136310; O- HUEFS 136672; P- HUEFS 136319; Q- HUEFS 136325; R- HUEFS 136112; S- HUEFS 136309). 
temente analisado. A espécie cresce associada a Anadyomene stellata, Boodlea composita, Bryopsis pennata, Chaetomorpha antennina (Bory) Kütz., Cladophora catenata, C. coelothrix Kütz., C. laetevirens (Dillwyn) Kütz., C. montagneana Kütz., C. vagabunda, Cladophora sp., Caulerpa fastigiata, Caulerpella ambigua, Codium intertextum Collins \& Herv., Dictyosphaeria versluysii, Ernodesmis verticillata (Kütz.) Børgesen, Halimeda opuntia (L.) J.V.Lamour., Ulva lactuca L., U. rigida C.Agardh, Ulva sp., Parvocaulis pusilla (M.Howe) S.Berger et al., Valonia aegagropila C.Agardh, Dictyopteris delicatula, Dictyota sp., Padina sp., Acanthophora muscoides, Amphiroa anastomosans, Ceramium dawsonii, Digenea simplex, Gelidiella acerosa, Hypnea musciformis, Jania adhaerens, Jania sp. e Palisada perforata. Epífita de Gelidiella acerosa. Epifitada por Ceramium sp., calcárias crostosas e diatomáceas.

\subsection{Phyllodictyon pulcherrimum J.E.Gray, J. Bot. 4: 70.} 1866.

Figuras 7 e 8 .

Talo verde-claro a verde-escuro, ereto, 7,5-10,5 cm alt. Fixo ao substrato por rizoides multicelulares, ramificados, originados na base do estipe. Estipe cilíndrico, ramificado ou não, com conspícuas constricções anelares na base. Ramificação inicial oposta formando um par de ramos laterais, originando lâminas separadas do eixo principal, posteriormente unidas umas às outras para formar uma única lâmina. Lâmina reticulada, cordiforme a ovalada, ramificada em um único plano, até $5 \mathrm{~cm}$ diâm., constituída por rede de filamentos anastomosados. Crescimento do talo exclusivamente por divisão celular apical, por invaginação da parede celular. Reforço estrutural do talo através de fixação de filamentos adjacentes por células tenaculares na extremidade apical das células. Célula apical cilíndrica com ápice arredondado, reto ou levemente curvado, 450-997-2300 $\mu \mathrm{m}$ compr. × 190-258-320 $\mu \mathrm{m}$ diâm.; células dos ramos terminais cilíndricas, 440-760-1000 $\mu \mathrm{m}$ compr. $\times 200-278-$ 378 um diâm.; células do eixo principal cilíndricas, 640-

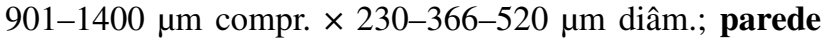
celular $2,5-10 \mu \mathrm{m}$ espessura. Inclusões cristalinas birrefringentes de oxalato de cálcio, em forma de hexágonos largos, visíveis no protoplasto.

Oceanos Atlântico e Índico (Guiry \& Guiry 2012). No litoral brasileiro, é referida apenas para os estados da Bahia e do Rio de Janeiro (Bravin et al. 1999; Yoneshigue-Valentin et al. 2006). H8/9: rara, possivelmente pela ausência de coletas em profundidades superiores a $4 \mathrm{~m}$. Talos férteis não observados.

Material examinado - Canavieiras, 30 mar. 2007, 35 m, A.M. Alves et al. s.n. (HUEFS 136330).

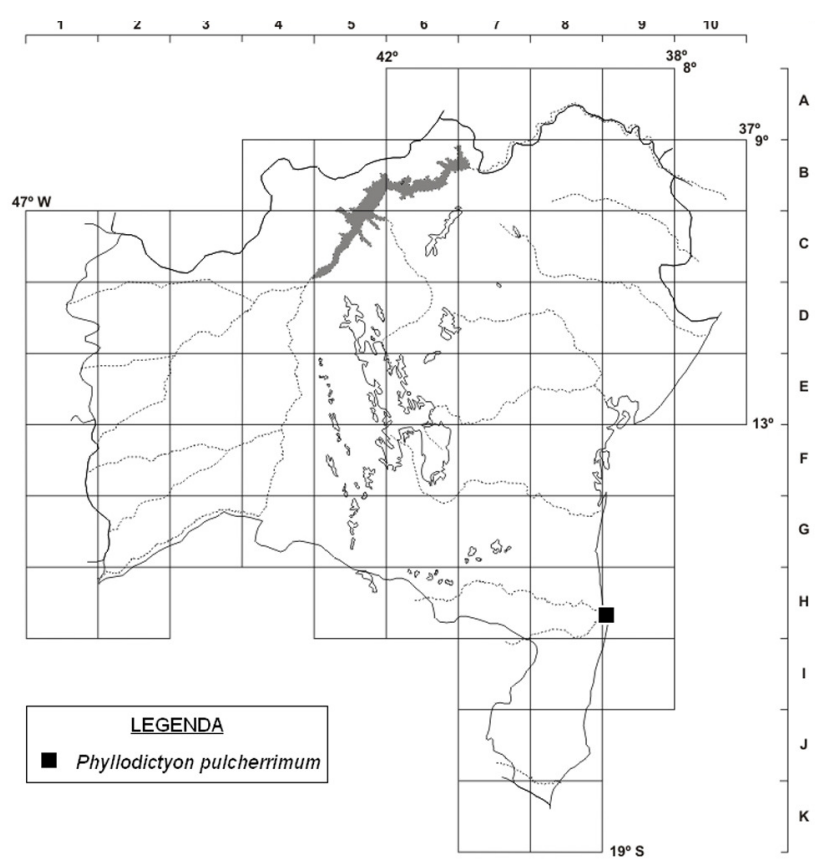

Figura 7. Mapa de distribuição de Phyllodictyon pulcherrimum no litoral do estado da Bahia.

Material adicional examinado - BRASIL. RIO DE JANEIRO: $22^{\circ} 23^{\prime} 18^{\prime \prime S}, 37^{\circ} 36^{\prime} 55^{\prime \prime} \mathrm{W}, 105$ m, 8 fev. 1997, Projeto REVIZEE s.n. (RFA 28714); ib., como "Struvea pulcherrima (J.E.Gray) Murray \& Boodle"; 22²3'16”S, 37³5'50"W, 22²3'16”'S, 37³5'18' 'W, Estação C1/1, 107 m, 8 fev. 1996, Projeto REVIZEE s.n. (RFA 28772).

Foi coletada em profundidade de $35 \mathrm{~m}$; a $310 \mathrm{~km}$ da costa norte do Rio de Janeiro (22²3'18'S e 37³6'55'W) e foi registrada a até $110 \mathrm{~m}$ (Bravin et al. 1999). Segundo Kraft \& Wynne (1996), a espécie é rara e exclusiva de águas profundas, tendo sido encontrada abaixo de $90 \mathrm{~m}$ de profundidade (Taylor 1960; Littler \& Littler 1997, 2000; Leliaert 2004).

\section{Agradecimentos}

Este trabalho é parte da dissertação de mestrado da primeira autora, desenvolvida no Programa de Pós-Graduação em Botânica da UEFS, com bolsa de mestrado da CAPES. Os autores agradecem à FAPESB pelo financiamento parcial do estudo (Proc. PPP 0011/2006), à UEFS (Projeto Flora da Bahia) e aos curadores dos herbários visitados e à Prof ${ }^{a}$ Dra. Yocie Yoneshigue Valentin (UFRJ) pela facilidade de acesso para análise do material estudado. 


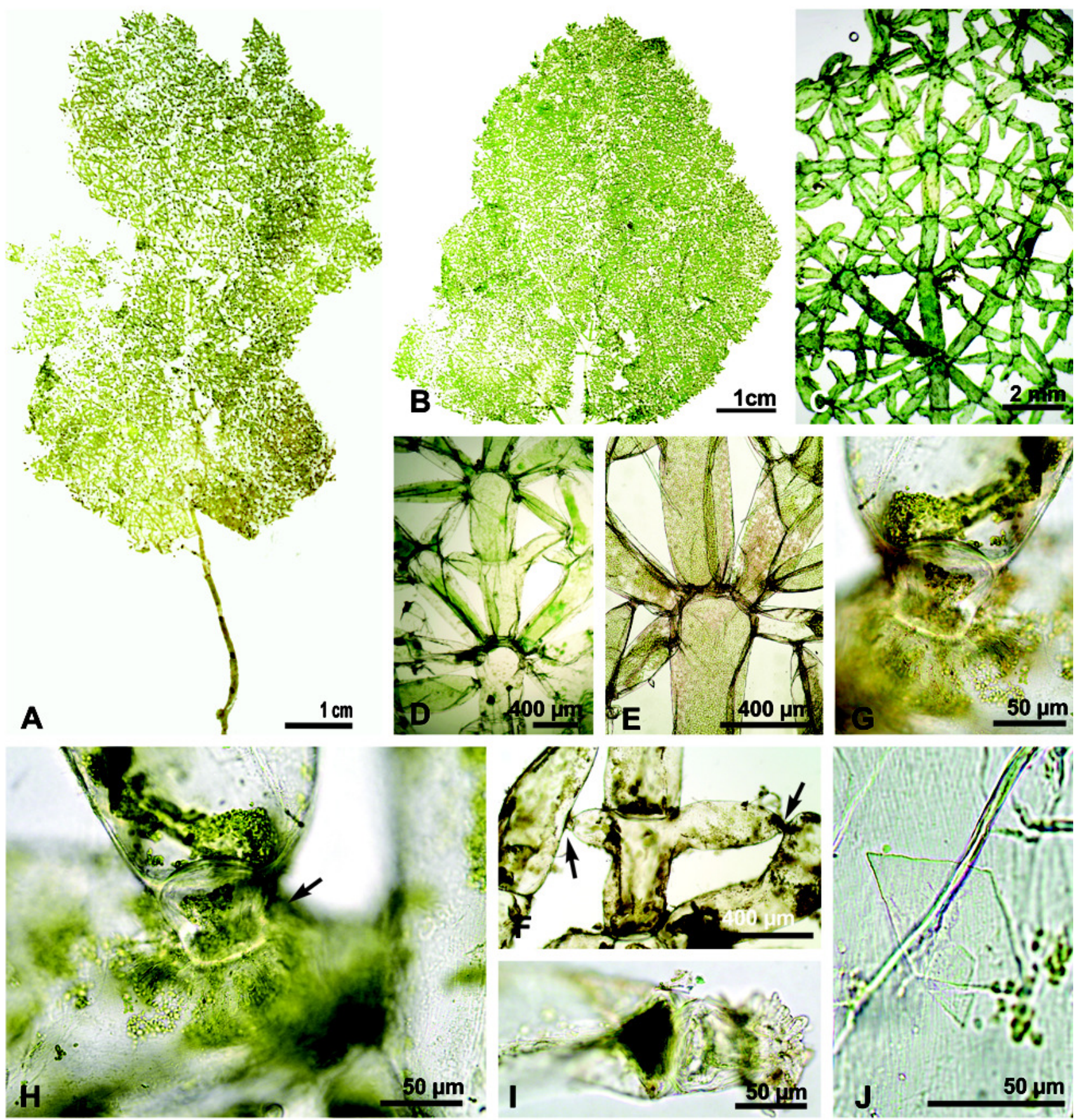

Figura 8. Phyllodictyon pulcherrimum: A- aspecto geral do talo; B- detalhe da lâmina; C- detalhe do padrão de ramificação, note eixo principal; D-Eramificação politômica; F- união de células adjacentes por células tenaculares; G-I- detalhes de células tenaculares; J- cristal de oxalato de cálcio em forma de hexágono largo (A, E, F, I, J- RFA 28714; B-D, G, H- RFA 28772).

\section{REFERÊNCIAS}

Alves, A.M.; Gestinari, L.M.S.; Andrade, N.A.; Almeida, W.A. \& Moura, C.W.N. 2012. Boodlea composita (Harv.) F.Brand (Chlorophyta) no litoral nordeste do Brasil. Acta Botanica Brasilica 26(2): 476-480.

Bravin, C.I.; Torres, J.; Gurgel, C.F.D. \& Yoneshigue-Valentin, Y. 1999. Novas ocorrências de clorofíceas marinhas de profundidade para o Brasil. Hoehnea 26: 121-133.

Guiry, M.D. \& Guiry, G.M. 2012. AlgaeBase. World-wide electronic publication, National University of Ireland, Galway. Disponível em <http://www.algaebase.org >; acesso em 1 out. 2012.

Kanagawa, A.I. 1984. Clorofíceas Marinhas Bentônicas do Estado da Paraíba - Brasil. Tese de Doutorado. Universidade de São Paulo.

Kraft, G.T. \& Wynne, M.J. 1996. Delineation of the genera Struvea Sonder and Phyllodictyon J.E. Gray (Cladophorales, 
Chlorophyta). Phycological Research 44: 129-143.

Leliaert, F. 2004. Taxonomic and Phylogenetic Studies in the Cladophorophyceae (Chlorophyta). Ph.D. Thesis. Ghent University.

Leliaert, F. \& Coppejans, E. 2006. A revision of Cladophoropsis Borgesen (Siphonocladales, Chlorophyta). Phycologia 49: 657-679.

Littler, D.S. \& Littler, M.M. 1997. An illustrated marine flora of the Pelican Cays, Belize. Bulletin of the Biological Society of Washington 9: 1-149.

Littler, D.S. \& Littler, M.M. 2000. Caribbean Reef Plants. An identification guide to the reef plants of the Caribbean, Bahamas, Florida and Gulf of Mexico. Offshore Graphics, Washington.

Moura, C.W.N.; Branco, C.Z.; Peres, C.K. \& Fajar, A. 2012. Ulvophyceae. In: Lista de Espécies da Flora do Brasil. Jardim Botânico do Rio de Janeiro. Disponível em http:// floradobrasil.jbrj.gov.br/2012/FB099035; acesso em 18 dez. 2012. 12

Muniz, J.A. 1993. Enumeração e novas ocorrências de algas marinhas bentônicas para o estado de Alagoas, Brasil. Revista Nordestina de Biologia 8(1): 1-4.

Nassar, C.A.G. 1994. An assessment to the benthic marine algae at Trindade Island, Espírito Santo, Brazil. Revista Brasileira de Biologia 54: 623-629.

Pedrini, A.G.; Ugadim, Y.; Braga, M.R.A. \& Pereira, S.M.B.
1992. Algas marinhas bentônicas do Arquipélago de Fernando de Noronha, Brasil. Boletim de Botânica da Universidade de São Paulo 13: 93-101.

Soares, P.L. \& Fujii, M.T. 2012. Epiphytic macroalgae from Boa Viagem Beach, Recife, Pernambuco State, Brasil. Check List 8(4): 662-665.

Széchy, M.T.M.; Maurat, M.C.S.; Nassar, C.A.G. \& Falcão, C. 1987. Adições à flora marinha bentônica do arquipélago de Fernando de Noronha. Nerítica 2: 135-146.

Taylor, W.R. 1960. Marine Algae of the Eastern Tropical and Subtropical Coasts of Americas. University of Michigan Press, Ann Arbor.

Wynne, M.J. 2011. A checklist of benthic marine algae of the tropical and subtropical western Atlantic: third Revision. Nova Hedwigia Beihefte 140: 1-166.

Yoneshigue, Y.; Boudouresque, C.F. \& Figueiredo, M.A.O. 1986. Flore algale marine de la Région de Cabo Frio, état de Rio de Janeiro (Brésil). 5 - Sur Boodlea composita (BoodleaceaeChlorophyta), Dictyota pardalis (Dictyotaceae-Phaeophyta) et Lophosiphonia cristata (Rhodomelaceae-Rhodophyta), espèces nouvelles pour la côte Brésilienne. Rickia 13: 17-27.

Yoneshigue-Valentin, Y.; Gestinari, L.M.S. \& Fernandes, D.R.P. 2006. Macroalgas. In: H.P. Lavrado \& B.L. Ignácio (eds), Biodiversidade Bentônica da Região Central da Zona Econômica Exclusiva Brasileira. Série Livros 18. Museu Nacional, Rio de Janeiro, p. 67-105. 13

\section{Lista de Exsicatas}

Almeida, W.R. s.n. HUEFS: 136665 (1.1); Alves, A.M. s.n. HUEFS: 136300, 136301, 136304, 136307, 136308 (1.1), $136309,136311,136312,136313,136314,136322,136328$ (3.1), 136330 (3.2), 136506, 136507, 136508, 136509, 136593, 136594, 136598, 136600, 136601, 136602, 136605, 136610, 136611, 136612, 136613, 136614, 136615, 136616, 136617, 136618 (2.1); Alves, G.L. s.n. HUEFS: 136507 (2.1); Boccanera, N.B. s.n. SP: 188251 (3.1), ALCB: 17291, 17425 (3.1); Joly, A.B. s.n. SPF: 82 (3.1); Martins, D.V. s.n. SP: 188254 (2.1), ALCB: 17030 (2.1), 17116, 17123 (3.1), 17136, 49341 (2.1); Minervino Netto, A. s.n. ALCB:
34634 (2.1); Moura, C.W.N. s.n. HUEFS: 136302, 136303, 136604, 136305, 136306 (1.1), 136310, 136315, 136316, 136317, 136318, 136319, 136321, 136323, 136324, 136325, 136326, 136327, 136329 (3.1), 136595, 136596, 136597, 136599, 136613, 136617 (2.1), 136672 (3.1); Nonato, E. s.n. SPF: 53079 (3.1); Nunes, A.P.M. s.n. ALCB: 22064 (2.1). Nunes, J.M.C. s.n. ALCB: 49251 (2.1); Projeto REVIZEE s.n. RFA: 28714, 28772 (3.2); Silva, S.A. s.n. ALCB: 036792 (2.1); Viana, L.T. s.n. ALCB: 17035 (3.1); Yoneshigue-Valentin, Y. s.n. RFA: 4010H, MOF 352, YY 2831 (1.1). 